\title{
DOUBLE SHOCK FRONTS FOR HYPERBOLIC SYSTEMS OF CONSERVATION LAWS IN MULTIDIMENSIONAL SPACE
}

\author{
AN TON BUI AND DENING LI
}

\begin{abstract}
The existence of a unique double shock front for hyperbolic systems of conservation laws in several space variables is established, extending an earlier result of Metivier. An example of a double shock wave arising from physical applications is given.
\end{abstract}

The purpose of this paper is to show the existence of a unique double shock front for hyperbolic systems of conservation laws in several space variables describing physical phenomena.

Unlike the one-dimensional case, it is only recently that Majda [7], using microlocal analysis, established the existence of a unique stable shock front for hyperbolic systems. For double shock fronts the only known result is due to Metivier [8,9], where $2 \times 2$ systems of conservation laws in two-dimensional space are considered.

In this paper the existence of a double shock front for hyperbolic systems of conservation laws in several space variables is established. When restricted to $2 \times 2$ systems in two-dimensional space, our result is similar to that of Metivier but our approach is somewhat different and perhaps simpler.

The notation and the basic assumptions are given in $\S 1$. The free-boundary problem is transformed in $\S 2$ into one with fixed boundaries. The linearized problem is considered in $\S 3$ and the nonlinear one is studied in $\S 4$, using the iteration method. Finally, in $\S 5$ an application to isentropic gas in two-dimensional space is given.

Let $G$ be an open subset of $R^{n}$ with a smooth boundary, $\partial G$, and let $x=\left(x_{1}, \ldots, x_{n}\right)$ be the generic point of $G$. For each $n$-tuple $\alpha=\left(\alpha_{1}, \ldots, \alpha_{n}\right)$ of nonnegative integers we write

$$
D^{\alpha}=\prod_{j=1}^{n} D_{j}^{\left(x_{j}\right.}, \quad D_{j}=\partial / \partial x_{j},|\alpha|=\sum_{j=1}^{n} \alpha_{j} .
$$

Received by the editors January 17, 1987 and, in revised form, April 15, 1988.

1980 Mathematics Subject Classification (1985 Revision). Primary 35L65, 35L50; Secondary $76 \mathrm{LO} 05$.

Work supported by NSERC (Canada). 
We denote by $H^{k}(G)$ the Sobolev space

$$
H^{k}(G)=\left\{u ; D^{\alpha} u \text { in } L^{2}(G),|\alpha| \leq k\right\} .
$$

It is a Hilbert space with the norm

$$
\|u\|_{H^{k}(G)}=\left\{\sum_{|\alpha| \leq k}\left\|D^{\alpha} u\right\|_{L^{2}(G)}^{2}\right\}^{1 / 2}
$$

and the corresponding inner product.

$H^{k-1 / 2}(\partial G)$ is the usual space of trace functions.

Let $\eta>1, S$ be a subset of $R_{t}$ and denote by $H_{\eta}^{k}(G \times S)$ the hyperbolic $\eta$-weighted Sobolev space $H_{\eta}^{k}(G \times S)=\left\{u(x, t): \eta^{k_{1}}\left(D^{\alpha} D_{t}^{k_{2}-\alpha} u\right) \exp (-\eta t)\right.$ in $\left.L^{2}(G \times S),|\alpha| \leq k_{2}, k_{1}+k_{2} \leq k\right\}$.

It is a Hilbert space with the norm

$$
\begin{aligned}
|u ; G \times S|_{k, \eta} & =\|u\|_{H_{\eta}^{k}(G \times S)} \\
& =\left\{\sum_{\substack{k_{1}+k_{2} \leq k \\
|\alpha| \leq k_{2}}} \int_{G \times S} \eta^{2 k_{1}}\left(D^{\alpha} D_{t}^{k_{2}-\alpha} u\right)^{2} \exp (-2 \eta t) d x d t\right\}^{1 / 2} .
\end{aligned}
$$

$H_{\eta}^{k}(\partial G \times S)$ is similarly defined with the norm

$$
|u ; \partial G \times S|_{k, \eta}=\|u\|_{H_{\eta}^{k}(\partial G \times S)} .
$$

In this paper we shall consider the system

$$
D_{t}\left\{F_{0}(u)\right\}+\sum_{j=1}^{n} D_{j}\left\{F_{j}(u)\right\}=0 \quad \text { in } R^{n} \times R_{t}^{+},
$$

where $u=\left(u_{1}, \ldots, u_{m}\right)$. The system (1.1) may be rewritten as

$$
D_{t} u+\sum_{j=1}^{n} A_{j}(u) D_{j} u=0 \quad \text { in } R^{n} \times R_{t}^{+} .
$$

Throughout the paper it is implicitly assumed that the system (1.2) is symmetric hyperbolic; i.e., there is a smoothly varying positive matrix $A_{0}(u)$ with $A_{0} A_{j}$ symmetric and $A_{j} \in C^{\infty}(Q)$ for $1 \leq j \leq n$, where $Q$ is the value domain of $u$ in $R^{m}$.

Let $\Gamma_{0}$ be a smooth hypersurface containing the origin and consider the Cauchy problem

$$
\left\{\begin{array}{l}
D_{t}\left\{F_{0}(u)\right\}+\sum_{j=1}^{n} D_{j}\left\{F_{j}(u)\right\}=0 \text { in } R^{n} \times R_{t}^{+}, \\
u(x, 0)=u_{0}(x) \text { in } R^{n},
\end{array}\right.
$$

where $u_{0}(x)$ is a piecewise smooth function with a discontinuity of the first kind along $\Gamma_{0}$. 
Since the speed of propagation of a hyperbolic system is finite and we are seeking a local solution of (1.3), there is no loss of generality in assuming that outside a neighborhood of the origin, $u_{0}=$ constant and $\Gamma_{0}$ is represented by $x_{1}=0$. In the neighborhood $G$ of the origin, we assume that $\Gamma_{0}$ is given by

$$
x_{1}=\phi_{0}\left(x_{2}, \ldots, x_{n}\right)=\phi_{0}\left(x^{\prime}\right) \text {. }
$$

Set

$$
\Omega_{0}^{+}=\left\{x: x \in G, \phi_{0}\left(x^{\prime}\right)<x_{1}\right\}, \quad \Omega_{0}^{-}=\left\{x: x \in G, x_{1}<\phi_{0}\left(x^{\prime}\right)\right\} .
$$

Let $\Gamma_{j}(t), j=1,2$, be two hypersurfaces given by $x_{1}=\phi_{j}\left(x^{\prime}, t\right)$ and let

$$
\begin{gathered}
\Omega^{1}(t)=\left\{x: x_{1}<\phi_{1}\left(x^{\prime}, t\right)\right\}, \quad \Omega^{2}(t)=\left\{x: \phi_{1}<x_{1}<\phi_{2}\right\}, \quad \text { and } \\
\Omega^{3}(t)=\left\{x: \phi_{2}\left(x^{\prime}, t\right)<x_{1}\right\} .
\end{gathered}
$$

Denote by

$$
\Omega_{T}^{i}=\bigcup_{0 \leq t \leq T} \Omega^{i}(t), \quad 1 \leq i \leq 3 .
$$

Definition 1.1. Let $\phi_{0}, u_{0}^{ \pm}$be $C^{\infty}$-functions with $\phi_{0}=0, u_{0}^{ \pm}=$const. for $|x|$ large. Then $\left\{u ; \Gamma_{1}(t), \Gamma_{2}(t)\right\}$ is said to be an $\mathscr{H}_{T}^{k}$-double shock wave solution of (1.3) if

(i) $\Gamma_{1}(0)=\Gamma_{2}(0)=\Gamma_{0}, u(x, 0)=u_{0}=\left\{u_{0}^{+}, u_{0}^{-}\right\}$;

(ii) $\Gamma_{j}(t)$ is given by

$$
x_{1}=\phi_{j}\left(t, x^{\prime}\right), \quad j=1,2,
$$

with $\phi_{j}$ in $H^{k+1}\left(R^{n-1} \times(0, T)\right)$ for some $T>0$;

(iii) $u$ is a solution of (1.3) with $u \mid \Omega_{T}^{i}$ in $H^{k}\left(\Omega_{T}^{i}\right), 1 \leq i \leq 3$;

(iv) the Rankine-Hugoniot conditions are satisfied:

$$
N_{0}^{j}\left[F_{0}(u)\right]^{j}+\sum_{i=1}^{n} N_{i}^{j}\left[F_{i}(u)\right]^{j}=0 \quad \text { on } \Gamma_{j}(t), j=1,2,
$$

where $\left(N_{0}^{j}, N_{1}^{j}, \ldots, N_{n}^{j}\right)$ is the unit exterior normal vector to $\Gamma_{j}(t)$ and $[f]^{j}$ is the jump of $f$ across $\Gamma_{j}(t)$.

In this paper we shall establish the existence of an $\mathscr{H}_{T}^{k}$-double shock wave solution of (1.3). We shall need the following assumptions.

Assumption (I). (i) $F_{j}$ is in $C^{\infty}$ for $j=1, \ldots, n$ and the system (1.2) satisfies the block structure of Majda [6].

(ii) There are two scalar functions $n_{0}^{1}, n_{0}^{2}$ with $n_{0}^{2}>n_{0}^{1}$ and a state function $\tilde{u}$ defined on $\Gamma_{0}$, all in $H_{u l}^{\infty}$ (the uniformly local Sobolev space of Kato [3]) such that

$$
n_{0}^{i}\left[F_{0}(\hat{u})\right]^{i}-\left[F_{1}(\hat{u})\right]^{i}+\sum_{j=2}^{n}\left[F_{j}(\hat{u})\right]^{i} D_{j} \phi_{0}=0 \quad \text { at } t=0,
$$

$i=1,2$, where $\hat{u}=\left(u_{0}^{-}, \tilde{u}, u_{0}^{+}\right)$. 
(iii) Two constant state shock fronts $\left(u_{0}^{-}, \tilde{u}, n_{0}^{1}\right)$ and $\left(\tilde{u}, u_{0}^{+}, n_{0}^{2}\right)$ are separately uniformly stable in the sense of Majda [6].

A compatibility condition is required throughout the paper.

Assumption (II). The compatibility conditions arising from

$$
D_{t}\left\{F_{0}(u)\right\}+\sum_{j=1}^{n} D_{j}\left\{F_{j}(u)\right\}=0
$$

and from

$$
\left[F_{0}(u)\right]^{i} D_{t} \phi_{i}-\left[F_{1}(u)\right]^{i}+\sum_{j=2}^{n}\left[F_{j}(u)\right]^{i} D_{j} \phi_{i}=0, \quad i=1,2,
$$

are satisfied up to order $r$ with $r$ large.

Remark 1.1. (1) The compatibility condition (II) is equivalent to the requirement that $\lambda \geq \lambda_{0}$ in Theorem 6.11 of [9].

(2) For an $m \times m$ system of conservation laws, if we are seeking an $m$-shock wave solution, then the compatibility condition is not needed. One has $m \times m$ boundary conditions with $m \times m$ unknowns. If a smooth solution exists, then the compatibility condition is automatically satisfied.

(3) In this paper, with the Euler equations of polytropic gases in mind, Assumption (II) is needed. A calculation shows that the stable multi-shock front solution for polytropic gases can have at most two shocks emanating from a single discontinuity.

In this section we shall transform the problem of finding a double shock wave solution of (1.3) into one with fixed boundaries. It will be carried out in three steps.

Step 1. Set

$$
t=\tau, \quad y^{\prime}=x^{\prime}, \quad y_{1}=t\left\{x_{1}-\phi_{1}\left(x^{\prime}, t\right)\right\} /\left(\phi_{2}-\phi_{1}\right) .
$$

The transformation (2.1) has been used in [2] to treat rarefaction waves; see also [10].

With the transformation (2.1) the domains $\Omega^{j}(t)$ become respectively

$$
\begin{aligned}
& Y_{1}=R^{n-1} \times R_{t}^{+} \times R^{-}=\left\{(y, t): y_{1}<0, t>0\right\}, \\
& Y_{2}=R^{n-1} \times R_{t}^{+} \times\left\{y_{1}: 0<y_{1}<t\right\}, \quad \text { and } \\
& Y_{3}=R^{n-1} \times R_{t}^{+} \times\left\{y_{1}: 0<t<y_{1}\right\} .
\end{aligned}
$$

Moreover, $\partial / \partial t=\partial / \partial \tau+a_{1}(y, \tau) \partial / \partial y_{1}$ with

$$
\begin{aligned}
a_{1}(y, t)= & D_{t}\left\{t\left(x_{1}-\phi_{1}\right)\left(\phi_{2}-\phi_{1}\right)^{-1}\right\} \\
= & -t\left(\phi_{2}-\phi_{1}\right)^{-1} D_{t} \phi_{1}+y_{1} t^{-1}\left(\phi_{2}-\phi_{1}\right)^{-1} \\
& \times\left\{\phi_{2}-\phi_{1}-t D_{1}\left(\phi_{2}-\phi_{1}\right)\right\} .
\end{aligned}
$$


Proposition 2.1. Suppose that Assumption (I) is satisfied and that $\phi_{j}$ is in $H_{\mathrm{loc}}^{k+1}\left(R^{n-1} \times R_{t}^{+}\right)$. Then $a_{1}$ is in $H_{\mathrm{loc}}^{k}\left(R^{n} \times R_{t}^{+}\right)$for any $k$ with $k \geq k_{0}>$ $1+[(n+1) / 2]$.

Proof. For $k>1+[(n+1) / 2], H^{k}$ is an algebra. It is clear that we have only to consider the case when $t \rightarrow 0^{+}$. Since $\phi_{2}\left(x^{\prime}, 0\right)-\phi_{1}\left(x^{\prime}, 0\right)=0$, we have

$$
\phi_{2}\left(x^{\prime}, t\right)-\phi_{1}\left(x^{\prime}, t\right)=t \int_{0}^{1} D_{t}\left\{\phi_{2}-\phi_{1}\right\}\left(x^{\prime}, s t\right) d s .
$$

So

$$
\begin{aligned}
\lim _{t \rightarrow 0^{+}}\left\{\left(\phi_{2}\left(x^{\prime}, t\right)-\phi_{1}\left(x^{\prime}, t\right)\right) t^{-1}\right\} & =\int_{0}^{1} D_{t}\left\{\phi_{2}-\phi_{1}\right\}\left(x^{\prime}, 0\right) d s \\
& =\left(n_{0}^{2}-n_{1}^{0}\right)\left(x^{\prime}\right)>0
\end{aligned}
$$

by Assumption (I). Rewriting $a_{1}(y, t)$ as

$$
a_{1}(y, t)=-D_{t} \phi_{1}\left\{t\left(\phi_{2}-\phi_{1}\right)^{-1}\right\}+y_{1}\left(\phi_{2}-\phi_{1}\right) t^{-1} D_{t}\left\{t\left(\phi_{2}-\phi_{1}\right)^{-1}\right\}
$$

we get the proposition by taking into account (2.3) and the Banach algebra property of $H_{\mathrm{loc}}^{k}$.

With the transformation (2.1) we have

$$
\frac{\partial}{\partial y_{j}}\left(y_{1}\right)=a_{j}(y, t)=-t\left(\phi_{2}-\phi_{1}\right)^{-1} D_{j} \phi_{1}-y_{1} t\left(\phi_{2}-\phi_{1}\right)^{-1} D_{j}\left\{t^{-1}\left(\phi_{2}-\phi_{1}\right)\right\}
$$

for $2 \leq j \leq n$.

Proposition 2.2. Suppose the hypothesis of Proposition 2.1 are satisfied. Then $a_{j}$ is in $H_{\mathrm{loc}}^{k}\left(R^{n} \times R_{t}^{+}\right), 2 \leq j \leq n$.

Proof. Same as that of Proposition 2.1.

We now rewrite equation $(1.3)$ as

$$
D_{t} u^{i}+\tilde{A}_{1}\left(u^{i} ; \phi_{1}, \phi_{2}\right) D_{1} u^{i}+\sum_{2}^{n} A_{j}\left(u^{i}\right) D_{j} u^{i}=0 \quad \text { in } Y_{i}, 1 \leq i \leq 3,
$$

where

$$
\tilde{A}_{1}\left(u^{i} ; \phi_{1}, \phi_{2}\right)=A_{1}\left(u^{i}\right)+a_{1}\left(\phi_{1}, \phi_{2}\right)-\sum_{j=2}^{n} A_{j}\left(u^{i}\right) a_{j}\left(\phi_{1}, \phi_{2}\right) .
$$

Step 2. We shall now use the compatibility Assumption (II) to transform the problem into one with homogeneous initial data.

Let $\tilde{u}, n_{0}^{i}, i=1,2$, be as in Assumption (I). We can construct $u_{0}^{j}, \phi_{0}^{i}$ in $H^{r}, r$ large, such that

$$
v^{j}(y, t)=u^{j}-u_{0}^{j}, \quad \psi^{i}\left(y^{\prime}, t\right)=\phi_{i}-\phi_{0}^{i}
$$


have zero initial data. Equation (2.5) becomes

$$
\begin{aligned}
D_{t} v^{j} & +\tilde{A}_{1}\left(v^{j}+u_{0}^{j} ; \phi_{0}^{i}+\psi^{i}\right) D_{1} v^{i}+\sum_{k=2}^{n} A_{k}\left(v^{j}+u_{0}^{j}\right) D_{k}\left(v^{j}+u_{0}^{j}\right) \\
& +\widetilde{A}_{1}\left(v^{j}+u_{0}^{j} ; \phi_{0}^{i}+\psi^{i}\right) D_{1} u_{0}^{j}=-D_{t} u_{0}^{j}, \quad 1 \leq j \leq 3, i=1,2 .
\end{aligned}
$$

Using a Taylor's expansion for $\widetilde{A}_{1}, A_{k}$ we obtain

$$
\begin{aligned}
D_{t} v^{j} & +\tilde{A}_{j}\left(v^{j}+u_{0}^{j} ; \phi_{0}^{i}+\psi^{i}\right) D_{1} v^{j} \\
& +\sum_{k=2}^{n} A_{k}\left(v^{j}+u_{0}^{j}\right) D_{k} v^{j}+M_{j}\left(v^{j}+u_{0}^{j} ; \phi_{0}^{i}+\psi^{i}\right) v^{j} \\
& +\sum_{i=1}^{2}\left\{N_{i j}^{1}\left(v^{j}+u_{0}^{j} ; \phi_{0}^{i}+\psi^{i}\right) \psi^{i}+N_{i j}^{2}\left(v^{j}+u_{0}^{j} ; \phi_{0}^{i}+\psi^{i}\right) \nabla \psi^{i}\right\}=f^{j},
\end{aligned}
$$

where

$$
-f^{j}(y, t)=D_{t} u_{0}^{j}+\tilde{A}_{1}\left(u_{0}^{j} ; \phi_{0}^{i}\right) D_{1} u_{0}^{j}+\sum_{k=2}^{n} A_{k}\left(u_{0}^{j}\right) D_{k} u_{0}^{j}
$$

and $M_{j}, N_{i j}^{1}, N_{i j}^{2}$ can be determined by Cauchy integral remainder, the explicit form of which is of no consequence in the following discussion.

Let us note that $f^{j}$ depends only on $u_{0}^{j}$, $\phi_{0}^{i}$, i.e., on the given data. With $u_{0}^{j}$ in $H^{r+1}\left(R^{n} \times R_{t}^{+}\right), \phi_{0}^{i}$ in $H^{r+2}\left(R^{n-1} \times R_{t}^{+}\right)$for large $r>0$, we have $f^{j}$ in $H_{\mathrm{loc}}^{r}$.

From Assumption (II) we obtain

$$
D_{t}^{s} f^{j}(y, 0)=0, \quad 0 \leq s \leq r-1,1 \leq j \leq 3,
$$

with $r$ large.

From the Rankine-Hugoniot condition we have

$$
\begin{aligned}
& {\left[F_{0}\left(v^{i}+u_{0}^{i}\right)\right] D_{t} \psi^{i}+\left[F_{0}\left(v^{i}+u_{0}^{i}\right)\right] D_{t} \psi_{0}^{i}-\left[F_{1}\left(v^{i}+u_{0}^{i}\right)\right]} \\
& +\sum_{k=2}^{n}\left\{D_{k} \psi^{i}\right\}\left[F_{k}\left(v^{i}+u_{0}^{i}\right)\right]+\sum_{k=2}^{n} D_{k} \phi_{0}^{i}\left[F_{k}\left(v^{i}+u_{0}^{i}\right)\right]=0 \\
& \quad \text { for } y_{1}=(i-1) t, i=1,2 .
\end{aligned}
$$

Using the Taylor's expansion we rewrite (2.11) as

$$
\begin{aligned}
& b_{0}^{i}\left(v^{i}, v^{i+1} ; u_{0}^{i}, u_{0}^{i+1}\right) D_{t} \psi^{i}+\sum_{k=2}^{n} b_{k}^{i}\left(v^{i}, v^{i+1} ; u_{0}^{i}, u_{0}^{i+1}\right) D_{k} \psi^{i} \\
& \quad+B_{i}\left(v^{i}, v^{i+1} ; u_{0}^{i}, u_{0}^{i+1}, \phi_{0}^{i}\right)=g^{i},
\end{aligned}
$$

where $B_{i}$ is a linear function,

$$
\begin{aligned}
& b_{0}^{i}=\left[F_{0}\left(v^{i}+u_{0}^{i}\right)\right], \quad b_{k}^{i}=\left[F_{k}\left(v^{i}+u_{0}^{i}\right)\right], \quad \text { and } \\
& -g^{i}=D_{t} \phi_{0}^{i}\left[F_{0}\left(u_{0}^{i}\right)\right]-\left[F_{1}\left(u_{0}^{i}\right)\right]+\sum_{k=2}^{n}\left[F_{k}\left(u_{0}^{i}\right)\right] D_{k} \phi_{0}^{i} .
\end{aligned}
$$


It is clear that $q^{i}$ depend only on the given data and by Assumption (II):

$$
D_{t}^{s} g^{i}(\cdot, 0)=0, \quad 0 \leq s \leq r-1,
$$

for large $r$.

Step 3. We shall now use a singular transformation and reduce the original problem to that of fixed boundaries. Let

$$
\begin{gathered}
\tau=\log t ; \quad x^{\prime}=y^{\prime} ; \quad x_{1}=y_{1} \quad \text { for } y_{1}<0 \\
x_{1}=y_{1} t^{-1} \quad \text { for } 0 \leq y_{1}<t \\
x_{1}=y_{1}-t+1 \quad \text { if } t \leq y_{1} .
\end{gathered}
$$

Then

$Y_{1}=R^{n-1} \times R_{t}^{+} \times R^{-}$becomes $X_{1}=R^{n-1} \times R_{\tau} \times R^{-}$,

$Y_{2}=R^{n-1} \times R_{t}^{+} \times\left\{y_{1}: 0<y_{1}<t\right\} \quad$ is mapped into $X_{2}=R^{n-1} \times R_{\tau} \times(0,1)$,

$Y_{3}=R^{n-1} \times R_{t}^{+} \times\left\{y_{1}: t<y_{1}\right\}$ is transformed into $X_{3}=R^{n-1} \times R_{\tau} \times(1, \infty)$.

To simplify the notation we shall write $t$ for $\tau$ and set $v={ }^{t}\left(v^{1}, v^{2}, v^{3}\right)$ with $v^{j}$ defined on $X_{j}$.

From (2.8) we get

$$
D_{t} v+a(v, \psi) D_{1} v+\sum_{j=2}^{n} a_{j}(v) D_{j} v+M(v, \psi) v+N(v, \psi)=e^{t} f,
$$

where

$$
\begin{aligned}
& a(v, \psi)=\left(\begin{array}{ccc}
e^{t} \tilde{A}_{1}\left(v^{1} ; \psi^{1}, \psi^{2}\right) & 0 \\
0 & \tilde{A}_{1}-x_{1} I & \\
& & e^{t}\left(\tilde{A}_{1}-I\right)
\end{array}\right), \\
& a_{j}=\left(\begin{array}{ccc}
e^{t} A_{j}\left(v^{1}\right) & & 0 \\
& e^{t} A_{j}\left(v^{2}\right) & \\
0 & & e^{t} A_{j}\left(v^{3}\right)
\end{array}\right), \quad 2 \leq j \leq n, \\
& M(v, \psi)=\left(\begin{array}{ccc}
e^{t} M_{1} & & 0 \\
& e^{t} M_{2} & \\
0 & & e^{t} M_{3}
\end{array}\right),
\end{aligned}
$$

with

$$
N(v, \psi)=\left(\begin{array}{cc}
e^{t} \sum_{i=1}^{2}\left(N_{i 1}^{1} \psi^{i}+N_{i 1}^{2} \nabla \psi^{i}\right) & e^{t} \sum_{i=1}^{2}\left(N_{i 2}^{1} \psi^{i}+N_{i 2}^{2} \nabla \psi^{i}\right) \\
0 & e^{t} \sum_{i=1}^{2}\left(N_{i 3}^{1} \psi^{1}+N_{i 3}^{2} \nabla \psi^{i}\right)
\end{array}\right) .
$$

The boundary conditions are now

$$
\begin{aligned}
& b_{0}^{i}\left(v^{i}, v^{i+1}\right) D_{t} \psi^{i}+e^{t} \sum_{j=2}^{n} b_{j}^{i}\left(v^{i}, v^{i+1}\right) D_{j} \psi^{i}+e^{t} B_{i}\left(v^{i}, v^{i+1}\right)=e^{t} g^{i} \\
& \text { on } S_{i}=\left\{(x, t): x_{1}=i-1\right\} \text { for } i=1,2 .
\end{aligned}
$$


The problem (2.15), (2.17) will be studied in the next two sections. The crucial observation is that the boundaries are fixed and are uncoupled.

In this section we shall consider a linearized form of equations (2.15) and (2.17). Let $w^{j}$ be in $H^{k}\left(x_{j}\right), \chi^{i}$ be in $H^{k+1}\left(S_{i}\right)$ for $1 \leq j \leq 3$ and $i=1,2$.

We shall study the linear problem

$$
\left\{\begin{array}{l}
D_{t} v+a(w, \chi) D_{1} v+\sum_{j=2}^{n} a_{j}(w) D_{j} v+M(w, \chi) v+N(v ; \psi)=e^{t} f, \\
b_{0}^{i}\left(w^{i}, w^{i+1}\right) D_{t} \psi^{i}+e^{t} \sum_{j=2}^{n} b_{j}^{i}\left(w^{i}, w^{i+1}\right) D_{j} \psi^{i}+e^{t} B_{i}\left(v^{i}, v^{i+1}\right)=e^{t} g^{i} \\
\text { on } S_{i}, i=1,2 .
\end{array}\right.
$$

Since the speed of propagation of a hyperbolic system is finite, we may assume without loss of generality that the coefficients of the matrices $a, a_{j}, M, N$, $b_{k}^{i}, B_{i}$ are constants for large $|x|$.

First we consider the simpler problem where the lower-order terms in (3.1) are omitted:

$$
\left\{\begin{array}{l}
D_{t} v+a(w ; \chi) D_{1} v+\sum_{j=2}^{n} a_{j}(w) D_{j} v=e^{t} f, \\
b_{0}^{i}\left(w^{i}, w^{i+1}\right) D_{t} \psi^{i}+\sum_{j=2}^{n} e^{t} b_{j}^{i}\left(w^{i}, w^{i+1}\right) D_{j} \psi^{i}+e^{t} B_{i}\left(v^{i}, v^{i+1}, \chi^{i}\right)=e^{t} g^{i}, \\
\text { on } S_{i}, i=1,2 .
\end{array}\right.
$$

Proposition 3.1. Let $f^{j}, g^{i}$ be given by (2.9)-(2.10) and by (2.13), respectively. Then:

(i) $e^{t} f^{1}$ is in $H_{\eta}^{k}\left(R^{n-1} \times(-c, 0) \times(-\infty, T)\right)$,

(ii) $e^{t} f^{2}$ belongs to $H_{\eta}^{k}\left(R^{n-1} \times(0,1) \times(-\infty, T)\right)$,

(iii) $e^{t} f^{3}$ is in $H_{\eta}^{k}\left(R^{n-1} \times(1, c) \times(-\infty, T)\right)$,

(iv) $e^{t} g$ is in $H_{\eta}^{k}\left(R^{n-1} \times(-\infty, T)\right)$ for $0<c<\infty$.

Proof. With $\tau=\log t$, we have from (2.10) by writing $t$ for $\tau$ :

$$
e^{-\eta t} D_{t}^{k} f^{j}(y, t) \in L^{2}\left(X_{j}\right) \text {. }
$$

A simple calculation shows that $e^{t} f^{j}$ belongs to the stated spaces if $r=\eta+k$. Similarly for $e^{t} q^{i}$.

By using a partition of unity we shall now assume that $e^{t} f^{j}, e^{t} q^{i}$ are zero and the coefficients $a, a_{j}, M, N, b_{k}^{i}, B_{i}$ in (3.2) are all constant for large $\left|x_{1}\right|$.

Definition 3.1. The $\mathscr{H}_{\eta}^{k}$-double shock wave solution of (3.1) is said to be uniformly linearly stable if the linear problem (3.2) is well posed and its solution 
satisfies the energy estimate

$$
\eta|v|_{0, \eta}^{2}+|v|_{0, \eta}^{2}+|\psi|_{1, \eta}^{2} \leq C\left\{\eta^{-1}\left|e^{t} f\right|_{0, \eta}^{2}+\sum_{i=1}^{2}\left|e^{t} g^{i}\right|_{0, \eta}^{2}\right\}
$$

for $\eta>\eta_{0}>0$ where $v=\left(v^{1}, v^{2}, v^{3}\right), \psi=\left(\psi^{1}, \psi^{2}\right)$, and

$$
\begin{gathered}
|v|_{0, \eta}^{2}=\sum_{j=1}^{3} \int_{X_{j}}\left(v^{j}\right)^{2} \exp (-2 \eta t) d x d t, \\
|v|_{0, \eta}^{2}=\sum_{i=1}^{2} \int_{S_{i}}\left\{\left(v^{i}\right)^{2}+\left(v^{i+1}\right)^{2}\right\} \exp (-2 \eta t) d x^{\prime} d t
\end{gathered}
$$

with

$$
|\psi|_{1, \eta}^{2}=\sum_{i=1}^{2} \sum_{\substack{k_{1}+k_{2} \leq 1 \\|\alpha| \leq k_{2}}} \int_{S_{i}} \eta^{2} k_{1}\left(D_{x^{\prime}}^{\alpha} D_{t}^{k_{2}-\alpha} \psi^{i}\right)^{2} \exp (-2 \eta t) d x^{\prime} d t .
$$

The boundaries in (3.2) are uncoupled; we now consider the problem separately near $S_{1}$ and near $S_{2}$. Set

$$
\begin{gathered}
\tilde{v}=\left(v^{1}, v^{2}\right)^{t}, \quad \tilde{f}=\left(f^{1}, f^{2}\right)^{t}, \\
\tilde{a}(x, t)=\left(\begin{array}{cc}
e^{t} \tilde{A}_{1} & 0 \\
0 & \tilde{A}_{1}-x_{1} t
\end{array}\right), \quad \tilde{a}_{j}=\left(\begin{array}{cc}
e^{t} A_{j} & 0 \\
0 & e^{t} A_{j}
\end{array}\right) .
\end{gathered}
$$

We have

$$
\left\{\begin{array}{l}
D_{t} \tilde{v}+\tilde{a} D_{1} \tilde{v}+\sum_{j=2}^{n} \tilde{a}_{j} D_{j} \tilde{v}=e^{t} \tilde{f}, \\
b_{0}^{1} D_{t} \psi^{1}+e^{t} \sum_{j=2}^{n} b_{j}^{1} D_{j} \psi^{1}+e^{t} B_{1}\left(v^{1}, v^{2}\right)=e^{t} g^{1} \text { on } S_{1} .
\end{array}\right.
$$

Extending the coefficients to be constant for large $\left|x_{1}\right|$ and making the change $x_{1} \mapsto-x_{1}$ in $X_{2}$, we obtain

$$
\left\{\begin{array}{l}
D_{t} \hat{v}+\hat{a} D_{1} \hat{v}+\sum_{j=2}^{n} \hat{a}_{j} D_{j} \hat{v}=e^{t} \hat{f} \quad \text { in } R^{n-1} \times R^{-} \times R_{t}, \\
b_{0}^{1} D_{t} \psi^{1}+\sum_{j=2}^{n} e^{t} b_{j}^{1} D_{j} \psi^{1}+e^{t} B_{1}\left(\hat{v}^{1}, \hat{v}^{2}\right)=e^{t} g^{1} \text { on } S_{1} .
\end{array}\right.
$$

Here $\hat{v}={ }^{t}\left(\hat{v}^{1}, \hat{v}^{2}\right), \hat{v}^{1}=v^{1}, \hat{v}^{2}\left(x_{1}, x^{\prime}, t\right)=v^{2}\left(-x_{1}, x^{\prime}, t\right)$.

For simplicity of notation we shall drop ${ }^{-}$when no confusion is possible.

In a similar fashion, we have

$$
\left\{\begin{array}{l}
D_{t} \bar{v}+\bar{a} D_{1} \bar{v}+\sum_{j=2}^{n} \bar{a}_{j} D_{j} \bar{v}=e^{t} \bar{f}, \\
b_{0}^{2} D_{t} \psi^{2}+\sum_{j=2}^{n} e^{t} b_{j}^{2} D_{j} \psi^{2}+e^{t} B_{2}\left(v^{2}, v^{3}\right)=e^{t} g^{2} \text { on } S_{2} .
\end{array}\right.
$$

The main result of the section is the following theorem. 
Theorem 3.1. Suppose that Assumptions (I), (II) are satisfied. Let $w^{j}$ be in $H^{k}\left(X_{j}\right), \chi^{i}$ be in $H^{k+1}\left(S_{i}\right)$ for $k \geq k_{0}>1+[(n+1) / 2]$, and $\left\|w^{2}\right\|_{H^{k}}+\left|\chi^{i}\right|_{H^{k+1}}<$ $\varepsilon_{0}$, for $\varepsilon_{0} \ll 1$. Then there exists a unique solution $\{v, \psi\}$ of $(3.1)$, satisfying

$$
\begin{array}{r}
\eta(((v)))_{k, \eta}^{2}+|v|_{k, \eta}^{2}+\eta\left|e^{-t} \psi\right|_{k, \eta}^{2}+(\nabla \psi)_{k, \eta}^{2} \\
\leq C\left\{\sum_{i=1}^{2}\left|g^{i}\right|_{k, \eta}^{2}+\eta^{-1} \sum_{j=1}^{3}\left|f^{j}\right|_{k, \eta}^{2}\right\},
\end{array}
$$

where

$$
\begin{gathered}
(((v)))_{k, \eta}^{2}=\left|e^{-t / 2} v^{1}\right|_{k, \eta}^{2}+\left|v^{2}\right|_{k, \eta}^{2}+\left|e^{-t / 2} v^{3}\right|_{k, \eta}^{2}, \\
(\nabla \psi)_{k, \eta}^{2}=\left|e^{-t} D_{t} \psi\right|_{k, \eta}^{2}+|D \psi|_{k, \eta}^{2} .
\end{gathered}
$$

$C$ is a constant depending only on $\left\|A_{j}(\cdot, w, \chi)\right\|_{H^{k}},\left\|b_{j}(\cdot, w)\right\|_{H^{k}}$.

First we shall study the problem (3.4).

Theorem 3.2. Suppose that the conditions in Theorem 3.1 are satisfied. Then there exist a unique $\left\{\hat{v}, \psi^{1}\right\}$, solution of (3.4). Moreover

$$
\begin{gathered}
\eta(((\hat{v})))_{k, \eta}^{2}+|\hat{v}|_{k, \eta}^{2}+\eta\left|e^{-1} \psi^{1}\right|_{k, \eta}^{2}+\left(\nabla \psi^{1}\right)_{k, \eta}^{2} \\
\leq C\left\{\left.g^{1}\right|_{k, \eta} ^{2}+\eta^{-1}\left(\left|f^{2}\right|_{k, \eta}^{2}+\left|f^{1}\right|_{k, \eta}^{2}\right)\right\},
\end{gathered}
$$

for $k \geq k_{0}>1+[(n+1) / 2]$.

$C$ depends on $\left\|A_{j}(\cdot, w, \chi)\right\|_{H^{k}},\left\|b_{j}(\cdot, w)\right\|_{H^{k}}$, and $\eta$ is a sufficiently large number.

Proof. (1) By hypothesis of separate stability at $(w, \chi)=0$, we have for $\varepsilon_{0} \ll 1$ :

$$
\left|b_{0}^{1} s+e^{t} \sum_{j=2}^{n} b_{j}^{1} \omega_{j}\right| \geq \gamma>0
$$

for $|s|^{2}+|\tilde{\omega}|^{2}=1$ with $s=i \xi+\eta, \eta>0$, and $\tilde{\omega}_{j}=e^{t} \omega_{j}, t$ negative.

Let $\Pi\left(D_{t}, e^{t} D_{j}\right)$ be the projection operator with symbol $\pi\left(x^{\prime}, t ; s, e^{t} \omega\right)$ given by

$$
\pi\left(x^{\prime}, t ; s, e^{t} \omega\right) w=w-\left(b_{0}^{1} s+e^{t} \sum_{j=2}^{n} b_{j}^{1} \omega_{j}\right) \frac{\left\langle b_{0}^{1} s+e^{t} \sum_{j=2}^{1} \omega_{j}, w\right\rangle}{\left|b_{0}^{1} s+e^{t} \sum_{j=2}^{n} b_{j}^{1} \omega_{j}\right|^{2}} .
$$

The symbol is clearly well defined for all $(x, t, \omega, s)$.

The boundary condition in (3.4) may be rewritten as

$$
\Pi\left(D_{t} e^{t} D_{j}\right) B_{1}(v)=\Pi\left(g_{1}\right)-\Pi\left(e^{t} b_{0}^{1} D_{t} \psi+\sum_{j=2}^{n} b_{j}^{1} D_{j} \psi\right) .
$$


(2) We shall now construct the Kreiss symmetrizer for (3.4), (3.6) by modifying Majda's proof [6]. Corresponding to $\tilde{A}_{1}, A_{j} e^{t}$, and $e^{t} b_{j}^{1}, 2 \leq j \leq n$, we construct a matrix-valued function $R\left(x, t ; s, e^{t} \omega\right)$ such that

(i) $R$ is Hermitian,

(ii)

$$
\begin{aligned}
\operatorname{Re}\left\{R a^{-1}\right. & \left.\left(s I+e^{t} \sum_{j=2}^{n} A_{j} \omega_{j}\right)\right\} \\
& =\operatorname{Re}\left\{R \tilde{A}_{1}^{-1}\left(s I+e^{t} \sum_{j=2}^{n} A_{j} \omega_{j}\right)\left(\begin{array}{cc}
e^{-t} I & 0 \\
0 & I
\end{array}\right)\right\} \\
& \geq \delta \eta \operatorname{diag}\left(e^{-t} I, I\right)
\end{aligned}
$$

for some $\delta>0$ and for $\eta \geq \eta_{0}>0$,

(iii)

$$
\begin{gathered}
R\left(x^{\prime}, t ; s, e^{t} \omega\right)+\delta_{*}^{-1}\left\{\Pi\left(x^{\prime}, t ; s, e^{t} \omega\right) B_{1}\left(x^{\prime}, t\right)\right\}^{*} \\
\times\left\{\Pi\left(x^{\prime}, t ; s, e^{t} \omega\right) B_{1}\left(x^{\prime}, t\right)\right\} \geq \delta_{*} I
\end{gathered}
$$

The positive constants $\delta$ and $\delta_{*}$ depend only on

$$
\left\|A_{r}(\cdot, w, \chi)\right\|_{H^{k}}, \quad\left\|b_{j}^{1}(\cdot, w)\right\|_{H^{k}} .
$$

Remark. Here the Kreiss symmetrizer $R$ is exactly the same as in [6], except that we have put $e^{t} \omega$ in the place of $\omega$ in [6]. This means that instead of constructing symmetrizer $R$ on the unit sphere $|s|^{2}+|\omega|^{2}=1$ and extending it as a homogeneous function of 0-degree outside $|s|^{2}+|\omega|^{2}=1$ as usual, now $R$ is homogeneous in $(s, \omega)$ only outside a larger and larger set $|s|^{2}+\left|e^{t} \omega\right|^{2} \leq 1$ as $t \rightarrow-\infty$. Consequently, the lower-order remainder terms coming from the composite or transpose of pseudodifferential operators may have norms which are uniformly bounded but no longer bounded by $C / \eta$.

This difficulty can be eliminated by noticing that in the following energy estimate, the operators $D_{j}(j=2, \ldots, n)$ are always accompanied by the factor $e^{t}$. Thus, the norm of the lower-order remainder terms can again be controlled by a small constant for $-t$ sufficiently large.

(3) We now establish the energy estimate. Consider

$$
\left(v, R a^{-1}\left(D_{t}+e^{t} \sum_{j=2}^{n} a_{j} D_{j}\right) v\right)=\left(v, R a^{-1}\left(e^{t} f\right)\right)-\left(v, R D_{1} v\right) .
$$

A simple calculation gives

$$
-2 \operatorname{Re}\left(v, R D_{1} v\right)=\operatorname{Re}\left(v,\left(R-R^{*}\right) D_{1} v\right)+\left(v, D_{1} R v\right)-\langle v, R v\rangle,
$$

where $\langle\cdot, \cdot\rangle$ is the inner product in $L^{2}\left(R^{n-1}\right)$ with $x_{1}=0$. 
Therefore:

$$
\begin{aligned}
& \operatorname{Re}\left\{\left(v, R a^{-1}\left(D_{t}+e^{t} \sum_{j=2}^{n} a_{j} D_{j}\right) v\right)\right\} \\
& =\operatorname{Re}\left\{\frac{1}{2}\left(v,\left(R-R^{*}\right) D_{1} v\right)\right. \\
& \left.\quad+\frac{1}{2}\left(v,\left(D_{1} R\right) v\right)-\frac{1}{2}\langle v, R v\rangle+\left(v, R a^{-1}\left(e^{t} f\right)\right)\right\} .
\end{aligned}
$$

By Lemma 4.2 of [6] and the techniques in $[1,4,5], R a^{-1}$ is an operator of order 0 . So

$$
\left|\operatorname{Re}\left(v, \operatorname{Ra}^{-1}\left(e^{t} f\right)\right)\right| \leq c\left\{\left|v^{1}\right|_{0, \eta}\left|e^{t} f^{1}\right|_{0, \eta}+\left|v^{2}\right|_{0, \eta}\left|e^{t} f^{2}\right|_{0, \eta} .\right.
$$

Similarly, $R-R^{*}$ is an operator of order -1 .

We have

$$
D_{1} v=a^{-1}\left(e^{t} f\right)-a^{-1}\left(D_{t} v\right)-\sum_{j=2}^{n} a^{-1} a_{j} D_{j} v .
$$

Taking into account the definition of $a^{-1}$ and of $a_{j}$ and noting that $t<0$, we obtain

$$
\begin{aligned}
\left|\operatorname{Re}\left(v_{1}\left(R-R^{*}\right) D_{1} v\right)\right| \leq & C\left|v^{1} e^{-t / 2}\right|_{0, \eta}\left\{\left|f^{1}\right|_{0, \eta}+\left|v^{1} e^{-t / 2}\right|_{0, \eta}\right\} \\
& +C\left|v^{2}\right|_{0, \eta}\left\{\left|f^{2} e^{t}\right|_{0, \eta}+\left|v^{2}\right|_{0, \eta}\right\} \\
\leq & C(((v)))_{0, \eta}\left\{|f|_{0, \eta}+(((v)))_{0, \eta}\right\} .
\end{aligned}
$$

Similarly, for $\left(v, D_{1} R . v\right)$. Hence

$$
\begin{gathered}
\left|\operatorname{Re}\left(v, \operatorname{Ra}^{-1}\left(e^{t} f\right)\right)\right|+\left|\operatorname{Re}\left(v,\left(R-R^{*}\right) D_{1} v\right)\right|+\left|\operatorname{Re}\left(v, D_{1} R . v\right)\right| \\
\leq C(((v)))_{0, \eta}^{2}+C(((v)))_{0, \eta}|f|_{0, \eta} .
\end{gathered}
$$

(4) We now estimate $\langle v, R v\rangle$ by considering the pseudodifferential operator with symbol

$$
R\left(0, x^{\prime}, t ; s, \tilde{\omega}\right)+\delta_{*}^{-1}\left\{\Pi\left(x^{\prime}, t ; s, \tilde{\omega}\right) B_{1}\left(x^{\prime}, t\right)\right\}^{*}\left\{\Pi\left(x^{\prime}, t ; s, \tilde{\omega}\right) B_{1}\left(x^{\prime}, t\right)\right\} .
$$

With the properties of $R$ we get

$$
\operatorname{Re}\left\{\langle v, R v\rangle+\delta_{*}^{-1}\left\langle v,\left\{\Pi B_{1}\right\}^{*}\left\{\Pi B_{1}(v)\right\}\right\rangle\right\} \geq \delta_{*}|v|_{0, \eta^{*}}^{2}
$$

We estimate $\operatorname{Re}\left\langle v,\left(\Pi B_{1}\right)^{*}\left(\Pi B_{1}(v)\right)\right\rangle$ by considering the boundary condition associated with the symbol $\left\{\Pi\left(x^{\prime}, t ; s, \tilde{\omega}\right) B_{1}\left(x^{\prime}, t\right)\right\}$. Following [6, p. 60] and taking into account the results in $[1,4,5]$, we have

$$
\operatorname{Re}\langle v, R v\rangle \geq \delta_{*}|v|_{0, \eta}^{2}-C\left|g^{1}\right|_{0, \eta}^{2}-C\left|e^{-t} \psi\right|_{0, \eta}^{2} .
$$

A computation as in [6, pp. 61-62] gives

$$
\mu\left|e^{-t} \psi\right|_{0, \eta}^{2}+\left|e^{-t} D_{t} \psi\right|_{0, \eta}^{2}+\left|D_{x} \psi\right|_{0, \eta}^{2} \leq C\left\{\left|g^{1}\right|_{0, \eta}^{2}+|v|_{0, \eta}^{2}\right\}
$$


Using the formula for the composition as well as the adjoint formula together with the sharp Garding inequality, we have from (3.7)-(3.11) the estimate of the theorem for $k=0$.

For estimates in $H_{\eta}^{k}\left(R_{-}^{n} \times(-\infty, 0)\right)$ we proceed as usual with the tangential derivatives first. The estimates for the normal derivatives are then obtained by using the noncharacteristic property.

Once the estimates are established, the existence is trivial to prove. Indeed, the adjoint problem has the same estimate, and by a Hilbert space argument, the problem has a unique solution in the considered space.

Theorem 3.3. Suppose all the hypotheses of Theorem 3.2 are satisfied. Then there exists a unique $\{\hat{v}, \psi\}$ solution of the problem

$$
\left\{\begin{array}{l}
D_{t} \hat{v}+\hat{a} D_{1} \hat{v}+\sum_{j=2}^{n} \hat{a}_{j} D_{j} \hat{v}+M \hat{v}+N(\psi)=e^{t} \hat{f}, \\
b_{0}^{1} D_{t} \psi+e^{t} \sum_{j=2}^{n} b_{j}^{1} D_{j} \psi+e^{t} B_{1}(\hat{v})=e^{t} g^{1} \quad \text { on } S_{1} .
\end{array}\right.
$$

Moreover, $\{\hat{v}, \psi\}$ satisfies the energy estimate of Theorem 3.2. $M, N$ are as in (3.1).

Proof. To simplify the notation we shall drop - . Let $0 \leq \lambda \leq 1$ and consider the problem

$$
\left\{\begin{array}{l}
D_{t} v+a D_{1} v+\sum_{j=2}^{n} a_{j} D_{j} v+\lambda(M v+N(\psi))=e^{t} f, \\
b_{0}^{1} D_{t} \psi+e^{t} \sum_{j=2}^{n} b_{j}^{1} D_{j} \psi+e^{t} B_{1}(v)=e^{t} g^{1} \text { on } S_{1} .
\end{array}\right.
$$

Denote by $\Lambda$ the set $\Lambda=\{\lambda: 0 \leq \lambda \leq 1$, (3.12) has a unique solution satisfying the estimate of Theorem 3.2$\}$.

(i) $\Lambda$ is nonempty. Indeed, by Theorem 3.2 we have $\lambda=0$ in $\Lambda$.

(ii) $\Lambda$ is closed. Suppose that $\lambda_{n}$ is in $\Lambda$ and that $\lambda_{n} \rightarrow \lambda$.

Corresponding to $\lambda_{n}$, we have $\left\{v_{n}, \psi_{n}\right\}$.

$$
\text { Set: } \quad F_{n}=e^{t} f-\lambda_{n}\left\{M v_{n}+N\left(\psi_{n}\right)\right\} .
$$

Applying the estimate of Theorem 3.2 with $F_{n}$ instead of $e^{t} f$, we obtain by taking $\eta$ large and noting that $t>0$

$$
\eta\left\|\left|v_{n}\right|\right\|_{k, \eta}^{2}+\left.\nabla \psi_{n}\right|_{k, \eta} ^{2}+\eta\left|\psi_{n}\right|_{k, \eta}^{2} \leq C,
$$

$C$ independent of $n$.

From the weak compactness of the unit ball in a Hilbert space we get $\{v, \psi\}$, solution of (3.12) with the desired estimate. Hence $\Lambda$ is closed.

(iii) $\Lambda$ is open. The proof is standard, using a Neumann series.

Therefore $\Lambda=[0,1]$ and with $\lambda=1$, we get the theorem.

Proof of Theorem 3.1. We shall first establish the energy estimate. Let $\left\{\Phi_{n}\right\}$ be a finite partition of unity and set $v_{n}=\Psi_{n} v$ with $v=\left(v^{1}, v^{2}, v^{3}\right)$. For $n$ with $\operatorname{supp}\left(\Phi_{n}\right) \cap S_{1} \neq \varnothing$ and $\operatorname{supp}\left(\Phi_{n}\right) \cap S_{2}=\varnothing$, Theorem 3.3 gives the estimate for $\left\{v_{n}^{1}, v_{n}^{2}\right\}$ and $\psi_{n}^{1}$. 
For $q$ with $\operatorname{supp}\left(\Phi_{q}\right) \cap S_{2} \neq \varnothing$ and $\operatorname{supp}\left(\Phi_{q}\right) \cap S_{1}=\varnothing$, a proof exactly as that of Theorem 3.3 again gives us the estimate for $\left\{v_{q}^{2}, v_{q}^{3}\right\}$ and $\psi_{q}^{2}$.

We have only to establish the estimate for $q$ with $\operatorname{supp}\left(\Phi_{q}\right) \cap S_{1} \cap S_{2}=\varnothing$ and $\operatorname{supp}\left(\Phi_{q}\right) \subset\left\{x_{1}: 0<x_{1}<1\right\}$, i.e., interior estimates. And this is standard.

Combining everything and considering the adjoint problem, we obtain the stated result.

In this section we shall use the iteration method to show the existence of a unique solution $\left\{v^{1}, v^{2}, v^{3}, \psi^{1}, \psi^{2}\right\}$ of $(2.15)-(2.17)$ in a neighborhood of $\tau=-\infty$, i.e., for small $t>0$. As done in $\S 3$ we shall write $t$ for $\tau$ to simplify the notation

Theorem 4.1. Suppose that Assumptions (I) and (II) are satisfied. Then there exist:

(1) $T_{*} \ll-1$;

(2) a unique $\{v, \psi\}$, solution of $(2.15),(2.17)$ in $\left(-\infty, T_{*}\right)$. Moreover,

$$
\eta(((v)))_{k, \eta}^{2}+|v|_{k, \eta}^{2}+\eta\left|e^{-t} \psi\right|_{k, \eta}^{2}+(\nabla \psi)_{k, \eta}^{2} \leq \varepsilon_{0} .
$$

For $k \geq k_{0}>1+[(n+1) / 2], \eta$ is sufficiently large. $(((\cdot)))_{k, \eta}$ and $(\cdot)_{k, \eta}$ are as in Theorem 3.1 .

Proof. Let $\phi_{T}(t)$ be a $C^{\infty}(R)$-function with $\phi_{T}(t)=1$ for $t \leq T$ and $\phi_{T}(t)=$ 0 for $t \geq T+1$. Consider the system

$$
\begin{gathered}
D_{t} v_{k}+a\left(v_{k-1}, \psi_{k-1}\right) D_{1} v_{k}+\sum_{j=2}^{n} a_{j}\left(v_{k-1}\right) D_{j} v_{k} \\
+N\left(v_{k-1}, \psi_{k-1}\right) \nabla \psi_{k}+M\left(v_{k-1}, \psi_{k-1}\right) v_{k}=e^{t} f \phi_{T}, \\
b_{0}^{i}\left(v_{k-1}^{i}, v_{k-1}^{i+1}\right) D_{t} \psi_{k}^{i}+e^{t} \sum_{j=2}^{n} b_{j}^{i}\left(v_{k-1}^{i}, v_{k-1}^{i+1}\right) D_{j} \psi_{k}^{i} \\
+e^{t} B_{i}\left(v_{k}^{i}, v_{k}^{i+1} ; \psi_{k-1}\right)=e^{t} g^{i} \phi_{T}, \quad i=1,2,
\end{gathered}
$$

with $k=1,2, \ldots$.

(1) Let $\left\{v_{0}, \phi_{0}\right\}=0$; then it follows from Theorem 3.1 that there exists a unique $\left\{v_{1}, \phi_{1}\right\}$, solution of (4.1). Moreover,

$$
\begin{aligned}
\eta\left(\left(\left(v_{1}\right)\right)\right)_{k, \eta}^{2} & +\left|v_{1}\right|_{k, \eta}^{2}+\eta\left|e^{-t} \psi_{1}\right|_{k, \eta}^{2}+\left(\nabla \psi_{1}\right)_{k, \eta}^{2} \\
& \leq C\left\{\sum_{i=1}^{2}\left|\phi_{T} g^{i}\right|_{k, \eta}^{2}+\eta^{-1}\left|\phi_{T} f\right|_{k, \eta}^{2}\right\} .
\end{aligned}
$$

$C$ depends on $\left\|A_{j}(\cdot)\right\|_{H^{k}},\left\|b_{j}^{i}(\cdot)\right\|_{H^{k}}$ and is independent of $T$. Let $\varepsilon_{0}>0$; then with $\phi_{T}$ as above, it is clear that for $T\left(\varepsilon_{0}\right) \ll-1$, the right-hand side of (4.2) is less than $\varepsilon_{0}$ :

$$
\eta\left(\left(\left(v_{1}\right)\right)\right)_{k, \eta}^{2}+\left|v_{1}\right|_{k, \eta}^{2}+\eta\left|e^{-t} \psi_{1}\right|_{k, \eta}^{2}+\left(\nabla \psi_{1}\right)_{k, \eta}^{2} \leq \varepsilon_{0}
$$

for $t \leq T\left(\varepsilon_{0}\right)$. 
(2) We reapply Theorem 3.1 with $w=v_{1}, \chi=\psi_{1}$. The constant $C$ of Theorem 3.1 depends on

$$
\left\|A_{j}\left(\cdot ; v_{1}, \psi_{1}\right)\right\|_{H_{\mathrm{loc}}^{k}}, \quad\left\|b_{j}^{i}\left(\cdot ; v_{1}\right)\right\|_{H_{\mathrm{loc}}^{k}} .
$$

For $k>1+[(n+1) / 2], H_{\mathrm{loc}}^{k}$ is an algebra. Since $\left\{v_{1}, \psi_{1}\right\}$ satisfies (4.3), an easy computation gives

$$
C\left(\left\|A_{j}\left(\cdot ; v_{1}, \psi_{1}\right)\right\|_{H_{\mathrm{loc}}^{k}},\left\|b_{j}^{i}\left(\cdot, v_{1}\right)\right\|_{H_{\mathrm{loc}}^{k}}\right)=C_{1}\left(\left\|A_{j}(\cdot)\right\|_{H_{\mathrm{loc}}^{k}},\left\|b_{j}^{i}(\cdot)\right\|_{H_{\mathrm{loc}}^{k}}, \varepsilon_{0}\right) .
$$

Theorem 3.1 gives

$$
\begin{gathered}
\eta\left(\left(\left(v_{2}\right)\right)\right)_{k, \eta}^{2}+\left|v_{2}\right|_{k, \eta}^{2}+\eta \mid e^{-t} \psi_{2} \|_{k, \eta}^{2}+\left(\nabla \psi_{2}\right)_{k, \eta}^{2} \\
\leq C_{1}\left\{\eta^{-1}\left|\phi_{T} f\right|_{k, \eta}^{2}+\left|\phi_{T} q\right|_{k, \eta}^{2}\right\} .
\end{gathered}
$$

With the definition of $\phi_{T}$, there exists $T_{*}\left(\varepsilon_{0}, \eta\right) \ll-1$ such that

$$
C_{1}\left\{\eta^{-1}\left|\phi_{T_{*}} f\right|_{k, \eta}^{2}+\left|\phi_{T_{*}} g\right|_{k, \eta}^{2}\right\} \leq \varepsilon_{0} .
$$

Clearly $T_{*}$ does not depend on $v_{2}, \psi_{2}$.

By induction we get $\left\{v_{s}, \psi_{s}\right\}$, solution of (4.1) for all $s=1,2, \ldots$.

(3) It remains to show that the sequence converges strongly in the appropriate norms. We have

$$
\begin{aligned}
& D_{t}\left(v_{s+1}-v_{s}\right)+a\left(v_{s}, \psi_{s}\right) D_{1}\left(v_{s+1}-v_{s}\right) \\
& +\sum_{j=2}^{n} a_{j}\left(v_{s}\right) D_{j}\left(v_{s+1}-v_{s}\right)+M\left(v_{s}, \psi_{s}\right)\left(v_{s+1}-v_{s}\right) \\
& +N\left(v_{s}, \psi_{s}\right) \nabla\left(\psi_{s+1}-\psi_{s}\right) \\
& =e^{t} f \phi_{T_{*}}-\left\{D_{t} v_{s}+a\left(v_{s}, \psi_{s}\right) D_{1} v_{s}+\sum_{j=2}^{n} a_{j}\left(v_{s}\right) D_{j} v_{s}\right. \\
& \left.+M\left(v_{s}, \psi_{s}\right) v_{s}+N\left(v_{s}, \psi_{s}\right) \nabla \psi_{s}\right\} .
\end{aligned}
$$

Replacing $e^{t} f \phi_{T_{*}}$ by (4.10), we obtain

$$
\begin{gathered}
D_{t}\left(v_{s+1}-v_{s}\right)+a\left(v_{s}, \psi_{s}\right) D_{1}\left(v_{s+1}-v_{s}\right)+\sum_{j=2}^{n} a_{j}\left(v_{s}\right) D_{j}\left(v_{s+1}-v_{s}\right) \\
+M\left(v_{s}, \psi_{s}\right)\left(v_{s+1}-v_{s}\right)+N\left(v_{s}, \psi_{s}\right) \nabla\left(\psi_{s+1}-\psi_{s}\right)=F_{s}
\end{gathered}
$$

where

$$
\begin{aligned}
F_{s}= & \left\{a\left(v_{s-1}, \psi_{s-1}\right)-a\left(v_{s}, \psi_{s}\right)\right\} D_{1} v_{s}+\sum_{j=2}^{n}\left\{a_{j}\left(v_{s-1}\right)-a_{j}\left(v_{s}\right)\right\} D_{j} v_{s} \\
& +\left\{M\left(v_{s-1}, \psi_{s-1}\right)-M\left(v_{s}, \psi_{s}\right)\right\} v_{s}+\left\{N\left(v_{s-1}, \psi_{s-1}\right)-N\left(v_{s}, \psi_{s}\right)\right\} \nabla \psi_{s} .
\end{aligned}
$$


Similarly, for the boundary equations we get

$$
\begin{aligned}
& b_{0}^{i}\left(v_{s}\right) D_{t}\left(\psi_{s+1}^{i}-\psi_{s}^{i}\right)+e^{t} \sum_{j=2}^{n} b_{j}^{i}\left(v_{s}\right) D_{j}\left(\psi_{s+1}^{i}-\psi_{s}^{i}\right) \\
& +e^{t} B_{i}\left(v_{s+1}^{i}, v_{s+1}^{i+1} ; \psi_{s}^{i}\right)-e^{t} B_{i}\left(v_{s}^{i}, v_{s}^{i+1} ; \psi_{s}^{i}\right)=G_{s}^{i} .
\end{aligned}
$$

(4) We now estimate $F_{s}$ and $G_{s}^{i}$. Since $H^{k-1}$ is an algebra we have

$$
\begin{aligned}
\eta^{-1}\left|F_{s}\right|_{k-1, \eta}^{2}+ & \left|G_{s}\right|_{k-1, \eta}^{2} \leq C_{2}\left\{\left(\left(\left(v_{s}\right)\right)\right)_{k, \eta}^{2}+\left|e^{-t} \psi_{s}\right|_{k, \eta}^{2}\right\} \\
& \times\left\{\left(\left(\left(v_{s}-v_{s-1}\right)\right)\right)_{k-1, \eta}^{2}+\left|e^{-t}\left(\psi_{s}-\psi_{s-1}\right)\right|_{k-1, \eta}^{2}\right\} . \\
\leq & C_{2} \varepsilon_{0}\left\{\left(\left(\left(v_{s}-v_{s-1}\right)\right)\right)_{k-1, \eta}^{2}+\left|e^{-t}\left(\psi_{s}-\psi_{s-1}\right)\right|_{k-1, \eta}^{2}\right\} .
\end{aligned}
$$

$C_{2}$ is independent of $s$.

Applying the estimate of Theorem 3.1 we obtain

$$
\begin{aligned}
& \left(\left(\left(v_{s}-v_{s+1}\right)\right)\right)_{k-1, \eta}^{2}+\left|e^{-t}\left(\psi_{s}-\psi_{s+1}\right)\right|_{k-1, \eta}^{2} \\
& \quad \leq C_{1} C_{2} \varepsilon_{0}\left\{\left(\left(\left(v_{s}-v_{s-1}\right)\right)\right)_{k-1, \eta}^{2}+\left|e^{-t}\left(\psi_{s}-\psi_{s-1}\right)\right|_{k-1, \eta}^{2}\right\} .
\end{aligned}
$$

With $C_{1} C_{2} \varepsilon_{0} \leq 1 / 2$, we have

$$
\left\{v_{s}, \psi_{s}\right\}+\{v, \psi\} \quad \text { in } H_{\eta}^{k-1} \text { and weakly in } H_{\eta}^{k} \text {. }
$$

It is not difficult to check that $\{v, \psi\}$ is the unique solution of the problem.

In this section we shall give an example of a double shock wave arising from physical applications.

Consider the equations of isentropic gas in two-dimensional space:

$$
\left\{\begin{array}{l}
D_{t} \rho+\sum_{j=1}^{2} D_{j}\left(\rho v_{j}\right)=0, \\
D_{t}\left(\rho v_{i}\right)+\sum_{j=1}^{2} D_{j}\left\{\rho v_{i} v_{j}+\delta_{i j} p(\rho)\right\}=0 \text { in } R^{2} \times R_{t}^{+},
\end{array}\right.
$$

where $\rho$ is the density, $v=\left(v_{1}, v_{2}\right)$ is the velocity $p(\rho)$ is the pressure, and $\delta_{i j}$ is the Kronecker delta function.

We take the initial jump surface $\Gamma_{0}$ to be the line $x_{1}=0$ and the initial conditions are

$$
\left\{\begin{array}{l}
\rho(x, 0)=\rho^{+} \quad \text { if } x_{1}>0 \text { or } x_{1}<0 \\
v_{i}(x, 0)=v_{i}^{ \pm}, \quad i=1,2
\end{array}\right.
$$

where $\rho^{ \pm}, v_{i}^{ \pm}$are constants.

We look for double shock fronts of the form

$$
\Gamma^{ \pm}(t)=\left\{(x, t): x_{1}=t \lambda_{ \pm}\right\}
$$

Clearly $\Gamma^{ \pm}(0)=\Gamma_{0}=\left\{x_{1}: x_{1}=0\right\}$. 
Let $\{\tilde{v}, \tilde{\rho}\}$ be the constant state of Assumption (I). The Rankine-Hugoniot condition on $\Gamma^{-}(t)$ may be written as

$$
\left\{\begin{array}{l}
-\lambda_{-}\left(\tilde{\rho}-\rho^{-}\right)+\left(\tilde{\rho} \tilde{v}_{1}-\rho^{-} v_{1}^{-}\right)=0, \\
-\lambda_{-}\left(\tilde{\rho} \tilde{v}_{1}-\rho^{-} v_{1}^{-}\right)+\left\{\tilde{\rho}\left(\tilde{v}_{1}\right)^{2}+p(\tilde{\rho})-\rho^{-}\left(v_{1}^{-}\right)^{2}-p\left(\rho^{-}\right)\right\}=0 \\
-\lambda_{-}\left(\tilde{\rho} \tilde{v}_{2}-\rho^{-} v_{2}^{-}\right)+\left\{\tilde{\rho} \tilde{v}_{1} \tilde{v}_{2}-\rho^{-} v_{1}^{-} v_{2}^{-}\right\}=0 .
\end{array}\right.
$$

The shock front is noncharacteristic and hence $\lambda_{-} \neq v_{1}^{-}, \lambda_{-} \neq \tilde{v}_{1}$. It follows that $\tilde{v}_{2}=v_{2}^{-}$. Thus, on $\Gamma^{-}(t)$ we have

$$
\left\{\begin{array}{l}
-\lambda_{-}\left(\tilde{\rho}-\rho^{-}\right)+\left(\tilde{\rho} \tilde{v}_{1}-\rho^{-} v_{1}^{-}\right)=0, \\
-\lambda_{-}\left(\tilde{\rho} \tilde{v}_{1}-\rho^{-} v_{1}^{-}\right)+\left\{\tilde{\rho}\left(\tilde{v}_{1}\right)^{2}+p(\tilde{\rho})-\rho^{-}\left(v_{1}^{-}\right)^{2}-p\left(\rho^{-}\right)\right\}=0, \\
\tilde{v}_{2}=v_{2}^{-} .
\end{array}\right.
$$

Similarly, on $\Gamma^{+}(t)$ we get

$$
\begin{aligned}
& \lambda_{+}\left(\tilde{\rho}-\rho^{+}\right)+\left(\tilde{\rho} \tilde{v}_{1}-\rho^{+} v_{1}^{+}\right)=0 \\
& \lambda_{+}\left(\tilde{\rho} \tilde{v}_{1}-\rho^{+} v_{1}^{+}\right)+\left\{\tilde{\rho}\left(\tilde{v}_{1}\right)^{2}+p(\tilde{\rho})-\rho^{+}\left(v_{1}^{+}\right)^{2}-p\left(\rho^{+}\right)\right\}=0 \\
& \tilde{v}_{2}=v_{2}^{+} .
\end{aligned}
$$

For polytropic gas, $p(\rho)=A \rho^{\gamma}, \gamma>1$, it is known that the uniform stability condition of the shock front in the sense of Majda is equivalent to that of compressibility, i.e., $\tilde{\rho}>\rho_{+}, \tilde{\rho}>\rho_{-}$. On the other hand, uniform stability is equivalent to the uniform well-posedness of the problem.

In view of Theorems 3.1, 4.1 and of Remark 3.1 it suffices to show the compressibility.

Let us consider the special symmetric case:

$$
\rho^{+}=\rho^{-}=\rho, \quad v_{1}=v_{1}^{-}=-v_{1}^{+}>0, \quad \lambda_{+}=\lambda, \quad \lambda_{-}=-\lambda .
$$

Then (5.4), (5.5) become

$$
\left\{\begin{array}{l}
-\lambda(\tilde{\rho}-\rho)+\rho v_{1}=0 \\
\lambda\left(\rho v_{1}\right)+\rho v_{1}^{2}+A \rho^{\gamma}-A(\tilde{p})^{\gamma}=0 .
\end{array}\right.
$$

Therefore

$$
(\tilde{\rho}-\rho)\left\{A(\tilde{\rho})^{\gamma}-A \rho^{\gamma}-\rho v_{1}^{2}\right\}=\left(\rho v_{1}\right)^{2}>0 .
$$

Equation (5.7) has a unique solution $\tilde{\rho}>\rho$. With $\tilde{\rho}$ known, (5.6) gives $\lambda$ and hence we obtain the two shock fronts $\Gamma^{ \pm}(t)$.

In the above example the constant state $\{\tilde{v}, \tilde{\rho}\}$ of Assumption (I) is given by $\tilde{v}_{1}=0, \tilde{v}_{2}=v_{2}^{-}=v_{2}^{+}$and $\tilde{\rho}$ is the unique solution of (5.7). As for Assumption (II), we have two relations for the two unknowns $\left(\lambda_{+}, \tilde{\rho}\right)$ and hence the compatibility condition is always satisfied. 


\section{REFERENCES}

1. M. Beals and M. C. Reed, Microlocal regularity theorems for nonsmooth pseudodifferential operators and applications to nonlinear problems, Trans. Amer. Math. Soc. 285 (1984), 159184.

2. E. Harabetian, A convergent expansion for hyperbolic systems of conservation laws, Trans. Amer. Math. Soc. 294 (1986), 383-424.

3. T. Kato, The Cauchy problem for quasilinear symmetric hyperbolic systems, Arch. Rational Mech. Anal. 58 (1975), 181-205.

4. Dening $\mathrm{Li}$, The nonlinear initial-boundary value problem and the existence of multidimensional shock wave for quasilinear hyperbolic-parabolic coupled systems, Chinese Ann. Math. 8B (1987), 252-280.

5. _ The $L^{2}$-boundedness of pseudo-differential operators with nonsmooth coefficient symbols, (preprint).

6. A. Majda, The stability of multidimensional shock fronts-a new problem for linear hyperbolic equations, Mem. Amer. Math. Soc. 273 (1983).

7. _ The existence of multidimensional shock fronts, Mem. Amer. Math. Soc. 281 (1983).

8. G. Metivier, Interaction de chocs, Sem. Bony-Sjöstrand-Meyer (1984-1985).

9. __ Interaction de deux chocs pour un systeme de deux lois de conservation, en dimension deux d'espace, Trans. Amer. Math. Soc. 296 (1986), 431-479.

10. S. Alinhac, Existence d'ondes de raréfaction pour des systèmes quasi-linéaires hyperboliques multidimensionnels (preprint).

Department of Mathematics, University of British Columbia, Vancouver, British Columbia, Canada

Department of Mathematics and Mechanics, Nanjing Institute of Technology, NanJing, People's Republic of China 